\title{
Lipid Metabolic Process
}

National Cancer Institute

\section{Source}

National Cancer Institute. Lipid Metabolic Process. NCI Thesaurus. Code C19405.

Anabolic and catabolic biochemical changes to lipids within a cell as materials needed for important life processes. 